\title{
RISK AND PROTECTIVE FACTORS FOR ANXIETY IMPACTING ACADEMIC PERFORMANCE IN POST-SECONDARY STUDENTS
}

\author{
KONRADT. LISNYJ \\ UNIVERSITY OF GUELPH
}

\author{
REGANRUSSELL \\ UNIVERSITY OF GUELPH
}

ANDREW PAPADOPOULOS

UNIVERSITY OF GUELPH

\begin{abstract}
This survey study measured the association between risk and protective factors of anxiety and its implications on the academic performance of 1,053 students at a four-year, public post-secondary institution in southwestern Ontario. Logistic regression analyses revealed 13 significant variables at the univariable level, while the multivariable model yielded seven significant factors. Students who felt hopeless significantly increased their odds of reporting anxiety adversely affecting their academic performance, while being able to manage daily responsibilities was the only protective factor against anxiety impacting students' educational attainment. By planning, designing, and implementing proactive programs focusing on these predictor variables, such interventions can equip students against the debilitative influence of anxiety on their academic success.
\end{abstract}

Keywords: anxiety, academic performance, post-secondary students, student wellness, risk factors, protective factors

\section{Résumé}

Cette enquête a mesuré les liens entre les facteurs de risque et les facteurs de protection de l'anxiété et leurs répercussions sur le rendement scolaire de 1053 étudiants d'un établissement postsecondaire public offrant des programmes de quatre ans du sud-ouest de l'Ontario. Une analyse de régression logistique univariée a révélé treize variables significatives, tandis que l'analyse multivariée en a révélé sept. Les étudiants qui se sentaient désespérés étaient beaucoup plus susceptibles de répondre que l'anxiété nuisait à leur rendement scolaire, tandis que la capacité de gérer leurs responsabilités quotidiennes était le seul facteur de protection contre l'anxiété affectant leur rendement. La planification, la conception et la mise en place de programmes proactifs axés sur ces variables explicatives peuvent aider les étudiants à contrer les effets néfastes de l'anxiété sur leur réussite scolaire.

Mots-clés : anxiété, rendement scolaire, bien-être des étudiants, étudiants de niveau postsecondaire, facteurs de risque, facteurs de protection

\section{Introduction}

Anxiety is the most prominent mental health problem impacting students pursuing higher education (Center for Collegiate Mental Health, 2018). The American College Health Association (ACHA) (ACHA, 2013a, 2019) found the rate of Canadian post-secondary students diagnosed with an anxiety disorder in the past 12 months has increased from $12.3 \%$ in 2013 to $23.7 \%$ in 2019. The ACHA (2019) also found $68.9 \%$ of Canadian post-secondary students reported experiencing overwhelming anxiety within the last year. This is problematic as anxiety is associated with memory, concentration, and cognitive function decline, which can contribute to poor academic performance (Afolayan et al., 2013; Kitzrow, 2009). This statement holds true as $34.6 \%$ of Canadian post-secondary students reported anxiety negatively impacted their school performance (ACHA, 2019).

The purpose of this study was to measure the association of various risk and protective factors in predicting students' reporting of anxiety impacting their academic performance, with the aim of developing proactive interventions at post-secondary institutions. Such information is integral in providing evidence for appropriately 
planning, designing, and implementing interventions that reduce the burden of anxiety and its implications on the academic success of tertiary students. This promotes a proactive approach to determine how to best plan wellness programs at institutions to prevent anxiety from unduly impacting students' academic achievement as well as their lives.

\section{Literature Review}

This literature review details the factors contributing to the growing prevalence of anxiety among post-secondary students, describes the effects of anxiety on student learning, identifies risk and protective factors associated with anxiety within higher education settings, and provides support concerning post-secondary institutions as an ideal setting for mental health promotion.

\section{Prevalence of Anxiety in Post-Secondary Students}

Anxiety rates have significantly increased in recent years, specifically in post-secondary student populations (Glauser, 2017; Marcotte \& Lévesque, 2018). The emerging adulthood (Arnett, 2004) stage of life, when most students pursue post-secondary education, is correlated with the age of peak onset for mental health problems, including anxiety (Reavley \& Jorm, 2010). Further, transitioning to post-secondary education brings upon increased competing academic, social, and financial demands, which increases student stress levels (Hunt \& Eisenberg, 2010; MacKean, 2011). Students' experiences of prolonged periods of stress also contribute to adverse mental health outcomes, including anxiety (Beiter et al., 2015; Moylan et al., 2013). It is important to note this increase in frequency and severity of mental health concerns is exhibited at both the undergraduate and graduate levels (Wyatt \& Oswalt, 2013). This growth can also partly be attributed to more students pursuing higher education, particularly those with pre-existing mental illnesses (MacKean, 2011). A final plausible explanation is the increased utilization of help-seeking behaviours among students to seek professional help for their mental health symptoms, thereby contributing to increased anxiety diagnoses (Hunt \& Eisenberg, 2010).

\section{Impacts of Anxiety Among Higher Education Students}

The implications of anxiety are both a critical public health and socioeconomic issue that warrant further scientific examination (Marcotte \& Lévesque, 2018), as anxiety is associated with several demographic (Farrer et al., 2016), psychological (Goswami et al., 2012), behavioural (Rose et al., 2015), social (Corona et al., 2017), financial (Eisenberg et al., 2007), and physical health implications (Adams et al., 2008) influencing post-secondary students' quality of life (Barrera \& Norton, 2009). In addition to these adverse effects, there is an extensive body of literature documenting the effects of anxiety impairing cognitive performance. Several research teams (e.g., Dull et al., 2015; Eysenck \& Derakshan, 2011; Eysenck et al., 2007; Merino et al., 2016) characterize anxiety as repetitive, intrusive, worrisome thoughts that both obstruct and consume the processing and storage capacity of an individual's working memory. Anxiety is further described as a maladaptive component of the learning process that impairs individuals' attentional control, even when there is no threatening stimulus present. As a result of interfering with memory and concentration, anxiety negatively impacts students' academic outcomes, namely poor academic performance and student attrition (Afolayan et al., 2013; Eisenberg et al., 2009; Kitzrow, 2009).

\section{Risk and Protective Factors Related to Anxiety Within Academic Settings}

Comparatively, fewer studies have conducted multivariate analyses to examine the interrelationship between several risk and protective factors of anxiety impacting academic performance (Larson et al., 2016). Conducting multivariate analyses are critical to understanding the relationship among variables to predict changes in the dependent variable (Larson et al., 2016).

Larson et al. (2016) argue student health and academic success are inextricably related, yet most studies use bivariate analyses to examine the relationship among these concepts. For instance, extensive research has identified the risk factors contributing to and the protective factors preventing against anxiety in post-secondary students (e.g., Esmaeelzadeh et al., 2018; Marcotte \& Lévesque, 2018), as well as the factors impacting 
academic performance (Brown et al., 2008; Chemers et al., 2001; Hartmann \& Prichard, 2018), individually.

Insufficient sleep is among the most reported risk factors impacting students' anxiety levels and their academic performance (Lund et al., 2010; Orzech et al., 2011). Dahl and Lewin (2002) indicate anxiety disorders interfere with individuals' ability to promote normal onset and continuity of sleep. Poor sleep quality is also associated with lower learning motivation, increased thought intrusions, and difficulty re-focusing on a task once distracted (Curcio et al., 2006; Lund et al., 2010). Other reported risk-taking factors related to increased anxiety and decreased academic performance, individually, include excessive alcohol consumption, low levels of physical activity, consuming energy-dense, nutrient-poor foods, engaging in risky sexual activities, feeling hopeless, and having perceptions of body dissatisfaction (Beiter et al., 2015; Böke et al., 2019; Kostanski \& Gullone, 1998; Snyder, 1999; Spring et al., 2012).

Protective factors for decreasing anxiety and improving academic performance, individually, commonly cited in the literature focus on promoting physical and mental well-being. This includes encouraging physical activity, having positive relationships, belonging to a community, living with a family member or friend, feeling hope, having life satisfaction, demonstrating effective time management skills, and recognizing one's own selfworth (e.g., Bellinger et al., 2015; Corona et al., 2017; Krick \& Sobal, 1990; Ranđelović et al., 2015).

\section{Post-Secondary Institutions as a Setting for Mental Health Promotion}

Educational institutions are responsible for promoting and supporting the optimal mental health and well-being of their students. Campuses with a supportive physical and social environment enable students to meet their long-term personal and professional goals, including psychosocial, financial, and physical benefits (Baum \& $\mathrm{Ma}, 2007)$. Accordingly, campuses are well-positioned as an ideal setting to identify, prevent, and intervene in mental health issues among this population (Reavley \& Jorm, 2010). However, campus mental health programs are often individualized and treatment-centered, where they lack a preventive focus. Such programs are unsustainable due to the influx in the number of students utilizing campus mental health services, particularly by those diagnosed with anxiety (Cornish et al., 2017; MacKean, 2011).

Instead, preventative approaches to promote positive mental health at the population level help mitigate mental health concerns (Evidence Exchange Network, 2016). This whole campus approach is fitting as anxiety is neither a linear nor a constant state (i.e., a non-anxious student can unexpectedly become an anxious student and vice versa), demonstrating that all students may find themselves in an anxious state at some point during their academic career. To better understand the factors that both promote and deter anxiety within academic settings, the researchers focused on proactive strategies to help students who are performing at a sub-optimal academic level, while also learning from those who experience anxiety but continue to perform at an optimal academic level.

\section{Method}

\section{Study Design and Data Collection}

The National College Health Assessment-II (NCHA-II) is a survey administered through the ACHA that collects information on North American post-secondary students' health habits, behaviours, and perceptions. The instrument has previously been evaluated for its reliability and validity (ACHA, 2013b). This cross-sectional survey study used data collected in Spring 2016 through the NCHA-II questionnaire at a four-year, English-speaking institution in southwestern Ontario, Canada. A random sample of enrolled students were invited by the ACHA via e-mail to participate in the web-based survey (a sample of the survey questionnaire is available online on the ACHA's website [ACHA, 2015]). The overall response proportion for this academic institution was $31.1 \%$. Approval from the institution's Research Ethics Board was received for this study (\#16FE031). Participants provided consent for their data to be used in this research.

\section{Outcome Variable}

The impact of anxiety on a student's academic performance was selected as the outcome of interest in this study. Students responded to the following survey question: "Within the last 12 months, has anxiety affected your academic performance?" Responses included six 
possible options: (a) did not experience this; (b) experienced, academics not affected; (c) lower grade on exam; (d) lower grade in course; (e) received an incomplete or dropped course; and (f) significant disruption to thesis. Responses were dichotomized into no (option b) and yes (options $c$ through $\mathrm{f}$ ) categories. It is important to note the effects of anxiety on all variations of academic performance were weighted equally (e.g., significant disruption to thesis was equal to receiving a lower grade in a course).

\section{Risk and Protective Factors}

The explanatory variables were selected a priori based on the risk and protective factors identified in the literature that were associated with anxiety and academic performance, individually. Questions were selected from the NCHA-Il survey that corresponded to the risk and protective factors of interest. The responses for most predictor variables were dichotomized, often into no and yes or less than half the time and more than half the time responses. Gender was one of four variables not dichotomized, in which there were three responses available: male, female, and other (encompassing transgender women, transgender men, genderqueer, and gender non-conforming individuals). The authors felt it was important to recognize the different experiences that cis- and transgender individuals might endure. Living arrangements had three possible responses: campus residence hall, parent and guardian's house, or other off-campus housing. Academic year was categorized by undergraduate years one through five and graduate. General health was also categorized to include three response options: above average, average, and below average. All of the predictor variables used in this survey study are found in Table 1.

Table 1. Outcome and explanatory variables

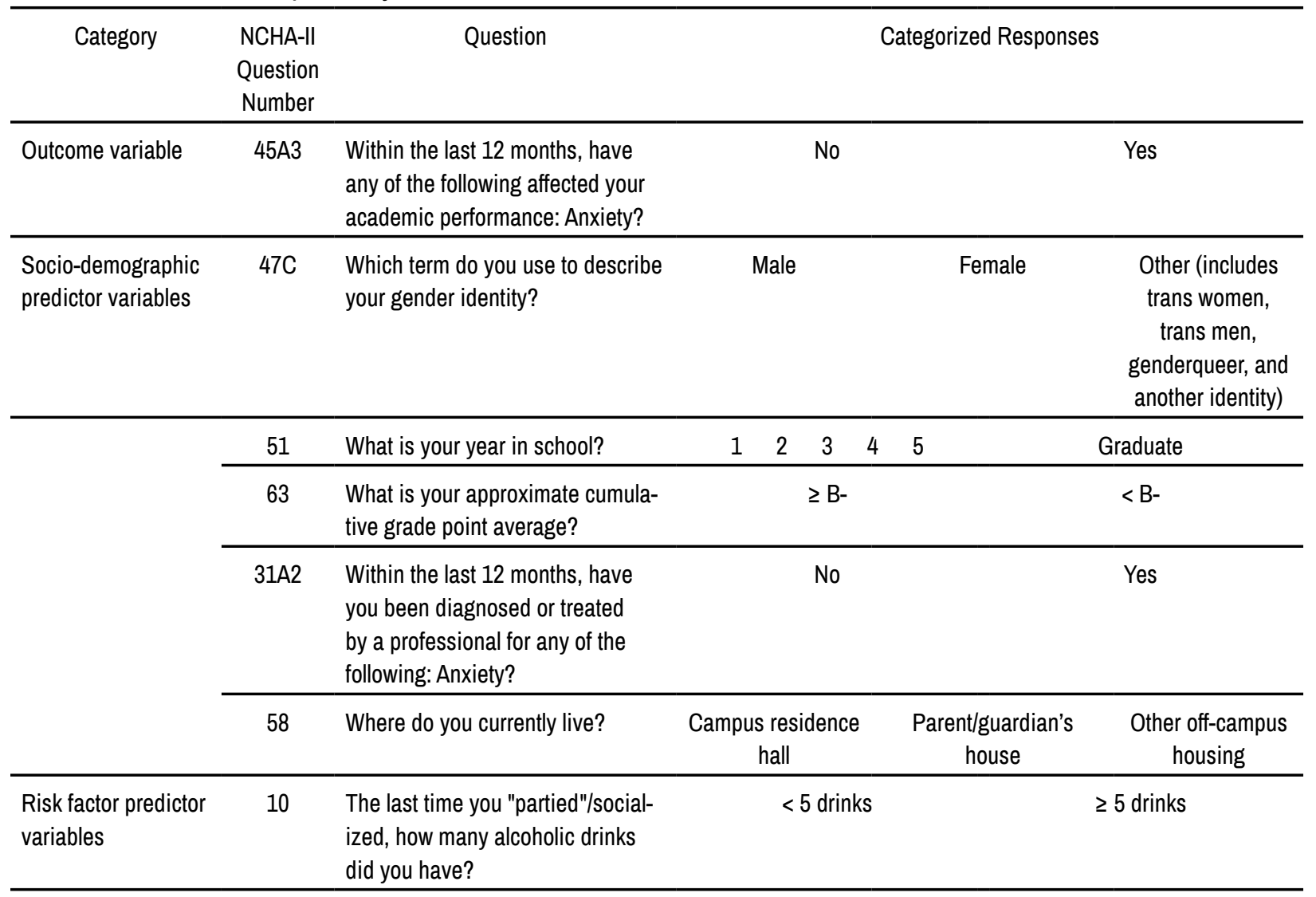




\begin{tabular}{|c|c|c|c|c|}
\hline \multirow[t]{7}{*}{ Category } & \multirow{2}{*}{$\begin{array}{l}\text { NCHA-II } \\
\text { Question } \\
\text { Number } \\
22 \mathrm{~B} / \mathrm{C}\end{array}$} & Question & \multicolumn{2}{|c|}{ Categorized Responses } \\
\hline & & $\begin{array}{l}\text { Within the last } 30 \text { days, how often } \\
\text { did you or your partner(s) use a } \\
\text { condom or other protective barrier } \\
\text { (e.g., male condom, female con- } \\
\text { dom, dam, glove) during vaginal } \\
\text { and anal intercourse? }\end{array}$ & $\begin{array}{l}\text { Unsafe sex (never, rarely, or } \\
\text { sometimes used protection) }\end{array}$ & $\begin{array}{l}\text { Safe sex (most of the time or } \\
\text { always used protection) }\end{array}$ \\
\hline & 28 & $\begin{array}{l}\text { How many servings of fruits and } \\
\text { vegetables do you usually have } \\
\text { per day? }\end{array}$ & $<5$ servings & $\geq 5$ servings \\
\hline & $29 \mathrm{~A}$ & $\begin{array}{l}\text { On how many of the past } 7 \text { days } \\
\text { did you do moderate intensity } \\
\text { cardio or aerobic exercise for at } \\
\text { least } 30 \text { minutes? }\end{array}$ & $\leq 5$ days & $>5$ days \\
\hline & $30 \mathrm{~A}$ & $\begin{array}{l}\text { Have you ever felt things were } \\
\text { hopeless? }\end{array}$ & No & Yes \\
\hline & 301 & $\begin{array}{l}\text { Within the last } 12 \text { months, has any } \\
\text { of the following been traumatic } \\
\text { or very difficult for you to handle: } \\
\text { Personal appearance? }\end{array}$ & No & Yes \\
\hline & $33 \mathrm{~K}$ & $\begin{array}{l}\text { Within the last } 12 \text { months, has any } \\
\text { of the following been traumatic } \\
\text { or very difficult for you to handle: } \\
\text { Sleep difficulties? }\end{array}$ & No & Yes \\
\hline \multirow[t]{6}{*}{$\begin{array}{l}\text { Protective factor } \\
\text { predictor variables }\end{array}$} & 1 & $\begin{array}{l}\text { How would you describe your } \\
\text { general health? }\end{array}$ & Above average & Below average \\
\hline & $3 \mathrm{~A} 3$ & $\begin{array}{l}\text { Are you interested in receiving } \\
\text { information on the following topics } \\
\text { from your college or university: } \\
\text { Depression/Anxiety? }\end{array}$ & No & Yes \\
\hline & 69A3 & $\begin{array}{l}\text { During the past month, how often } \\
\text { do you feel satisfied with life? }\end{array}$ & Less than half the time & More than half the time \\
\hline & 69A5 & $\begin{array}{l}\text { During the past month, how often } \\
\text { do you feel that you belonged to a } \\
\text { community (like a social group or } \\
\text { your neighborhood)? }\end{array}$ & Less than half the time & More than half the time \\
\hline & 69B3 & $\begin{array}{l}\text { During the past month, how often } \\
\text { do you feel good at managing the } \\
\text { responsibilities of your daily life? }\end{array}$ & Less than half the time & More than half the time \\
\hline & $69 \mathrm{~B} 4$ & $\begin{array}{l}\text { During the past month, how often } \\
\text { do you feel that you had warm and } \\
\text { trusting relationships with others? }\end{array}$ & Less than half the time & More than half the time \\
\hline
\end{tabular}




\section{Data Analysis}

All statistical analyses were performed using STATA/ SE 15.1 (StataCorp LLC, 2018). Descriptive statistics were conducted to review each variable in the dataset. A Spearman's rank correlation test was conducted to examine pairwise correlations. Any correlation greater than 0.7 was deemed collinear. Univariable logistic regression was performed using an alpha error level of $5 \%$ to evaluate unconditional associations between each individual predictor variable and the outcome of interest. Multivariable logistic regression was performed to model the factors associated with experiencing anxiety that impact academic performance. A manual backward-selection process was used to build this model. All factors were added to the model initially using a $p$-value of .05 , and removed one at a time based on the highest, non-significant $p$-value. As factors were removed, their impact on all other statistically significant coefficients were assessed to ensure that confounding variables remained in the model (using $\geq 30 \%$ change as a reference) (Dohoo et al., 2009). Two-way interaction terms were generated between all significant variables and tested within the model, with a $p$-value cut-off of $<.05$. A likelihood ratio test was used to compare each model to the previous one to ensure it fits the data better until all variables in the multivariable model had a significant $p$-value at the $5 \%$ significance level.

Model diagnostics were performed to ensure the model fit the data appropriately. A Pearson goodnessof-fit test (GOF) was conducted and deemed appropriate for the binomial data within the multivariable model. Covariate patterns with large leverage were noted and re-introduced into the model to check if there was a $\geq$ $30 \%$ change in coefficients or standard error in other variables. The models with and without the influential observations were compared using deviance, delta-beta, delta-chi-square, and delta-deviance plotted against leverage to determine if there was a significant difference in the GOF results between the models. If there was not a significant change in the GOF results, the covariate pattern was retained in the model.

\section{Results}

\section{Descriptive Statistics}

There were 1,053 respondents who answered the outcome variable: $60.0 \%$ indicated anxiety impacted their academic performance in the past year. The majority of respondents identified as female (79.3\%) and as undergraduate students (81.9\%). A very small percentage of respondents (1.0\%) identified as a gender other than male or female. Some students also reported receiving a diagnosis and/or treatment for anxiety (30.0\%), although females had a higher percentage of diagnosis than males (31.9\% versus $22.3 \%)$. Most respondents (80.5\%) reported a grade point average (GPA) greater than or equal to B-. Living arrangements varied, with the most common being other off-campus housing (67.7\%). Table 2 provides a breakdown of all risk and protective variables.

\section{Univariable Logistic Regression}

Univariable logistic regression analyses yielded 13 significant variables. Factors that increased the odds of reporting anxiety impacting students' academic performance included having a GPA below B- (odds ratio $(O R)=4.302)$, general health perceived as below average $(\mathrm{OR}=3.900)$ or average (OR=2.008), feeling hopeless (OR=3.391), experiencing sleep difficulties (OR=2.545), having negative perceptions of one's body image $(\mathrm{OR}=2.487)$, receiving a diagnosis or treatment for anxiety (OR=2.106), having an interest in receiving information about depression and/or anxiety (OR=1.916), and binge drinking ( $O R=1.322)$. On the other hand, effectively managing daily responsibilities $(\mathrm{OR}=0.362)$ and being satisfied with life $(\mathrm{OR}=0.408)$ lowered the odds of an individual's academic performance being impacted by anxiety. Having positive relationships with others also had a protective effect (OR=0.468), as did community integration ( $\mathrm{OR}=0.589)$ and being enrolled in a graduate degree program (OR=0.435).

\section{Multivariable Logistic Regression}

Seven variables were significant and remained in the multivariable logistic regression model based on the data of 948 respondents. The two factors with the highest OR were individuals reporting a GPA less 
Table 2. Descriptive statistics for outcome and explanatory variables

\begin{tabular}{|c|c|c|c|c|}
\hline & $\begin{array}{c}\text { Male } \\
n=207\end{array}$ & $\begin{array}{c}\text { Female } \\
n=833\end{array}$ & $\begin{array}{l}\text { Other } \\
n=10\end{array}$ & $\begin{array}{c}\text { All } \\
n=1,053\end{array}$ \\
\hline \multicolumn{5}{|c|}{ Anxiety impacted academic performance $(n=1,053)$} \\
\hline No $(n=421)$ & $45.4 \%$ & $38.8 \%$ & $30.0 \%$ & $40.0 \%$ \\
\hline Yes $(n=632)$ & $54.6 \%$ & $61.2 \%$ & $70.0 \%$ & $60.0 \%$ \\
\hline Gender $(n=1,050)$ & $19.7 \%$ & $79.3 \%$ & $1.0 \%$ & \\
\hline \multicolumn{5}{|l|}{ Academic year $(n=1,044)$} \\
\hline Undergraduate $(n=856)$ & $80.7 \%$ & $82.2 \%$ & $90.0 \%$ & $81.9 \%$ \\
\hline 1st year $(n=200)$ & $17.4 \%$ & $19.8 \%$ & $10.0 \%$ & $19.2 \%$ \\
\hline 2 nd year $(n=203)$ & $19.3 \%$ & $19.7 \%$ & $0.0 \%$ & $19.4 \%$ \\
\hline 3rd year $(n=215)$ & $18.8 \%$ & $20.6 \%$ & $40.0 \%$ & $20.5 \%$ \\
\hline 4th year $(n=174)$ & $16.9 \%$ & $16.5 \%$ & $30.0 \%$ & $16.7 \%$ \\
\hline 5 th year $(n=64)$ & $8.2 \%$ & $5.6 \%$ & $10.0 \%$ & $6.1 \%$ \\
\hline Graduate $(n=188)$ & $19.3 \%$ & $17.8 \%$ & $10.0 \%$ & $18.1 \%$ \\
\hline \multicolumn{5}{|l|}{ GPA $(n=1,039)$} \\
\hline$<$ B- $(n=202)$ & $23.2 \%$ & $18.3 \%$ & $40.0 \%$ & $19.5 \%$ \\
\hline$\geq$ B- $(n=837)$ & $76.8 \%$ & $81.7 \%$ & $60.0 \%$ & $80.5 \%$ \\
\hline \multicolumn{5}{|c|}{ Diagnosed with/treated for anxiety $(n=1,050)$} \\
\hline No $(n=734)$ & $77.7 \%$ & $68.1 \%$ & $70.0 \%$ & $70.0 \%$ \\
\hline Yes $(n=316)$ & $22.3 \%$ & $31.9 \%$ & $30.0 \%$ & $30.0 \%$ \\
\hline \multicolumn{5}{|l|}{ Living accommodation $(n=1,051)$} \\
\hline Campus residence ( $n=243)$ & $19.9 \%$ & $24.0 \%$ & $20.0 \%$ & $23.1 \%$ \\
\hline Parent or guardian's house $(n=96)$ & $8.7 \%$ & $9.3 \%$ & $0.0 \%$ & $9.2 \%$ \\
\hline Other off-campus housing ( $n=712)$ & $71.4 \%$ & $66.7 \%$ & $80.0 \%$ & $67.7 \%$ \\
\hline \multicolumn{5}{|l|}{ Alcohol consumption $(n=1,048)$} \\
\hline$<5$ drinks $(n=572)$ & $44.4 \%$ & $57.0 \%$ & $60.0 \%$ & $54.5 \%$ \\
\hline$\geq 5$ drinks $(n=476)$ & $55.6 \%$ & $43.0 \%$ & $40.0 \%$ & $45.5 \%$ \\
\hline \multicolumn{5}{|c|}{ Protection during sexual intercourse $(n=612)$} \\
\hline No $(n=300)$ & $46.0 \%$ & $50.1 \%$ & $20.0 \%$ & $49.0 \%$ \\
\hline Yes $(n=312)$ & $54.0 \%$ & $49.9 \%$ & $80.0 \%$ & $51.0 \%$ \\
\hline \multicolumn{5}{|l|}{ Servings of fruits/vegetables $(n=1,052)$} \\
\hline$<5$ servings $(n=924)$ & $92.8 \%$ & $86.8 \%$ & $90.0 \%$ & $88.0 \%$ \\
\hline$\geq 5$ servings $(n=128)$ & $7.2 \%$ & $13.2 \%$ & $10.0 \%$ & $12.0 \%$ \\
\hline
\end{tabular}




\begin{tabular}{|c|c|c|c|c|}
\hline & $\begin{array}{c}\text { Male } \\
n=207\end{array}$ & $\begin{array}{c}\text { Female } \\
n=833\end{array}$ & $\begin{array}{l}\text { Other } \\
n=10\end{array}$ & $\begin{array}{c}\text { All } \\
n=1,053\end{array}$ \\
\hline \multicolumn{5}{|c|}{ Exercise for at least 30 minutes $(n=1,048)$} \\
\hline$\leq 5$ days $(n=845)$ & $82.0 \%$ & $80.2 \%$ & $90.0 \%$ & $80.7 \%$ \\
\hline$>5$ days $(n=203)$ & $18.0 \%$ & $19.8 \%$ & $10.0 \%$ & $19.3 \%$ \\
\hline \multicolumn{5}{|l|}{ Feeling hopeless $(n=1,051)$} \\
\hline No $(n=246)$ & $30.0 \%$ & $21.9 \%$ & $20.0 \%$ & $23.5 \%$ \\
\hline Yes $(n=805)$ & $70.0 \%$ & $78.1 \%$ & $80.0 \%$ & $76.5 \%$ \\
\hline \multicolumn{5}{|c|}{ Negative perceptions of body image $(n=1,053)$} \\
\hline No $(n=607)$ & $64.7 \%$ & $56.2 \%$ & $50.0 \%$ & $57.8 \%$ \\
\hline Yes $(n=446)$ & $35.3 \%$ & $43.8 \%$ & $50.0 \%$ & $42.2 \%$ \\
\hline \multicolumn{5}{|l|}{ Sleep difficulties $(n=1,053)$} \\
\hline No $(n=584)$ & $56.5 \%$ & $55.7 \%$ & $30.0 \%$ & $55.6 \%$ \\
\hline Yes $(n=469)$ & $43.5 \%$ & $44.3 \%$ & $70.0 \%$ & $44.4 \%$ \\
\hline \multicolumn{5}{|l|}{ General health $(n=1,036)$} \\
\hline Above average $(n=394)$ & $46.4 \%$ & $36.3 \%$ & $20.0 \%$ & $38.1 \%$ \\
\hline Average $(n=436)$ & $34.3 \%$ & $44.2 \%$ & $20.0 \%$ & $42.0 \%$ \\
\hline Below average $(n=206)$ & $19.3 \%$ & $19.5 \%$ & $60.0 \%$ & $19.9 \%$ \\
\hline \multicolumn{5}{|l|}{ Information seeking $(n=1,010)$} \\
\hline No $(n=87)$ & $18.1 \%$ & $6.3 \%$ & $11.1 \%$ & $8.6 \%$ \\
\hline Yes $(n=923)$ & $81.9 \%$ & $93.7 \%$ & $88.9 \%$ & $91.4 \%$ \\
\hline \multicolumn{5}{|l|}{ Satisfied with life $(n=1,041)$} \\
\hline Less than half the time $(n=599)$ & $57.1 \%$ & $57.2 \%$ & $80.0 \%$ & $57.4 \%$ \\
\hline More than half the time $(n=442)$ & $42.9 \%$ & $42.3 \%$ & $20.0 \%$ & $42.6 \%$ \\
\hline \multicolumn{5}{|l|}{ Belonging to a community $(n=1,044)$} \\
\hline Less than half the time $(n=632)$ & $62.3 \%$ & $60.0 \%$ & $70.0 \%$ & $60.5 \%$ \\
\hline More than half the time $(n=412)$ & $37.7 \%$ & $40.0 \%$ & $30.0 \%$ & $39.5 \%$ \\
\hline \multicolumn{5}{|c|}{ Managing daily responsibilities $(n=1,046)$} \\
\hline Less than half the time $(n=671)$ & $64.0 \%$ & $63.8 \%$ & $80.0 \%$ & $64.0 \%$ \\
\hline More than half the time $(n=375)$ & $36.0 \%$ & $36.1 \%$ & $20.0 \%$ & $36.0 \%$ \\
\hline \multicolumn{5}{|c|}{ Positive relationships with others $(n=1,043)$} \\
\hline Less than half the time $(n=492)$ & $54.9 \%$ & $44.7 \%$ & $80.0 \%$ & $47.0 \%$ \\
\hline More than half the time $(n=551)$ & $45.1 \%$ & $55.3 \%$ & $20.0 \%$ & $53.0 \%$ \\
\hline
\end{tabular}


Table 3. Univariable logistic regression results

\begin{tabular}{|c|c|c|c|c|c|}
\hline \multirow[t]{2}{*}{ Variable } & \multirow{2}{*}{$\begin{array}{c}\text { Regression } \\
\text { Coefficient } \\
(\beta)\end{array}$} & \multirow[t]{2}{*}{$\begin{array}{l}\text { Odds Ratio } \\
\qquad(\mathrm{OR})\end{array}$} & \multirow[t]{2}{*}{$p$-value } & \multicolumn{2}{|c|}{$\begin{array}{c}\text { Odds Ratio (OR) } \\
95 \% \text { Confidence Interval }\end{array}$} \\
\hline & & & & Lower Limit & Upper Limit \\
\hline \multicolumn{6}{|l|}{ Gender } \\
\hline Female & Ref. & Ref. & & & \\
\hline Male & -0.273 & 0.761 & 0.082 & 0.560 & 1.035 \\
\hline Other & 0.391 & 1.478 & 0.573 & 0.379 & 5.756 \\
\hline \multicolumn{6}{|l|}{ Academic year } \\
\hline \multicolumn{6}{|l|}{ Undergraduate } \\
\hline 1st year & Ref. & Ref. & & & \\
\hline 2nd year & 0.089 & 1.093 & 0.673 & 0.723 & 1.654 \\
\hline 3rd year & -0.098 & 0.907 & 0.633 & 0.606 & 1.356 \\
\hline 4th year & -0.410 & 0.664 & 0.054 & 0.437 & 1.008 \\
\hline 5th year & -0.196 & 0.822 & 0.507 & 0.460 & 1.469 \\
\hline Graduate & -0.833 & 0.435 & $<0.001^{*}$ & 0.289 & 0.654 \\
\hline \multicolumn{6}{|l|}{ GPA } \\
\hline$\geq \mathrm{B}-$ & Ref. & Ref. & & & \\
\hline$<\mathrm{B}-$ & 1.459 & 4.302 & $<0.001^{*}$ & 2.891 & 6.395 \\
\hline \multicolumn{6}{|c|}{ Diagnosed with/treated for anxiety } \\
\hline No & Ref. & Ref. & & & \\
\hline Yes & 0.745 & 2.106 & $<0.001^{*}$ & 1.584 & 2.801 \\
\hline \multicolumn{6}{|l|}{ Living accommodations } \\
\hline Campus residence & Ref. & Ref. & & & \\
\hline Parent or guardian's house & -0.364 & 0.695 & 0.136 & 0.431 & 1.122 \\
\hline Other off-campus housing & -0.132 & 0.876 & 0.388 & 0.649 & 1.183 \\
\hline \multicolumn{6}{|l|}{ Alcohol consumption } \\
\hline$<5$ drinks & Ref. & Ref. & & & \\
\hline$\geq 5$ drinks & 0.279 & 1.322 & $0.029 *$ & 1.030 & 1.564 \\
\hline \multicolumn{6}{|c|}{ Protection during sexual intercourse } \\
\hline No & Ref. & Ref. & & & \\
\hline Yes & 0.107 & 1.113 & 0.515 & 0.806 & 1.536 \\
\hline \multicolumn{6}{|l|}{ Servings of fruits/vegetables } \\
\hline$<5$ servings & Ref. & Ref. & & & \\
\hline$\geq 5$ servings & 0.116 & 1.123 & 0.550 & 0.767 & 1.644 \\
\hline
\end{tabular}




\begin{tabular}{lcccc}
\hline Variable & $\begin{array}{c}\text { Regression } \\
\text { Coefficient } \\
(\beta)\end{array}$ & $\begin{array}{c}\text { Odds Ratio } \\
(\mathrm{OR})\end{array}$ & $\begin{array}{c}p \text {-value } \\
\text { Lower Limit }\end{array}$ & $\begin{array}{c}\text { Odds Ratio (OR) } \\
95 \% \text { Confidence Interval }\end{array}$ \\
\hline
\end{tabular}

Exercise for at least 30 minutes

\begin{tabular}{lccccc}
$\leq 5$ days & Ref. & Ref. & & & \\
$>5$ days & 0.050 & 1.051 & 0.753 & 0.768 & 1.439 \\
\hline Feeling hopeless & & & & & \\
No & Ref. & Ref. & & & \\
Yes & 1.221 & 3.391 & $<0.001^{*}$ & 2.520 & 4.565 \\
\hline
\end{tabular}

\begin{tabular}{|c|c|c|c|c|c|}
\hline Yes & 1.221 & 3.391 & $<0.001^{*}$ & 2.520 & 4.565 \\
\hline \multicolumn{6}{|c|}{ Negative perceptions of body image } \\
\hline No & Ref. & Ref. & & & \\
\hline Yes & 0.911 & 2.487 & $<0.001^{*}$ & 1.916 & 3.231 \\
\hline \multicolumn{6}{|l|}{ Sleep difficulties } \\
\hline No & Ref. & Ref. & & & \\
\hline Yes & 0.934 & 2.545 & $<0.001^{*}$ & 1.965 & 3.297 \\
\hline \multicolumn{6}{|l|}{ General health } \\
\hline Above average & Ref. & Ref. & & & \\
\hline Average & 0.697 & 2.008 & $<0.001^{*}$ & 1.521 & 2.652 \\
\hline Below average & 1.361 & 3.900 & $<0.001^{*}$ & 2.665 & 5.709 \\
\hline \multicolumn{6}{|l|}{ Information seeking } \\
\hline No & Ref. & Ref. & & & \\
\hline Yes & 0.650 & 1.916 & $0.004^{*}$ & 1.231 & 2.979 \\
\hline \multicolumn{6}{|l|}{ Satisfied with life } \\
\hline Less than half the time & Ref. & Ref. & & & \\
\hline More than half the time & -0.897 & 0.408 & $<0.001^{*}$ & 0.316 & 0.526 \\
\hline \multicolumn{6}{|l|}{ Belonging to a community } \\
\hline Less than half the time & Ref. & Ref. & & & \\
\hline More than half the time & -0.530 & 0.589 & $<0.001^{*}$ & 0.457 & 0.758 \\
\hline \multicolumn{6}{|c|}{ Managing daily responsibilities } \\
\hline Less than half the time & Ref. & Ref. & & & \\
\hline More than half the time & -1.017 & 0.362 & $<0.001^{*}$ & 0.279 & 0.470 \\
\hline \multicolumn{6}{|c|}{ Positive relationships with others } \\
\hline Less than half the time & Ref. & Ref. & & & \\
\hline More than half the time & -0.760 & 0.468 & $<0.001^{*}$ & 0.363 & 0.603 \\
\hline
\end{tabular}

Note: ${ }^{*} p \leq 0.05$ 
than B- (OR=3.102) and individuals who felt hopeless $(\mathrm{OR}=2.061)$. Self-reporting one's general health as below average $(\mathrm{OR}=1.800)$ or average $(\mathrm{OR}=1.501)$ also increased the odds of a student experiencing anxiety that impacted their academic performance. Other risk factors included sleep difficulties (OR=1.768), receiving a diagnosis or treatment for anxiety $(\mathrm{OR}=1.654)$, and having negative perceptions of body image (OR=1.413). Managing daily responsibilities $(\mathrm{OR}=0.562)$ was the only protective factor in the multivariable model. No confounding variables or significant interaction terms were identified.

\section{Model Diagnostics}

No issues of multicollinearity among predictor variables were found. A Pearson goodness-of-fit test was performed, and all results were reported as non-significant, demonstrating that the model fits the data. There were no significant outliers or covariate patterns that skewed the model.

\section{Discussion}

Below, this discussion highlights critical factors that can be addressed on post-secondary campuses to reduce

Table 4. Multivariable logistic regression results

\begin{tabular}{|c|c|c|c|c|c|}
\hline \multirow[t]{2}{*}{ Variable } & \multirow[t]{2}{*}{$\begin{array}{l}\text { Regression } \\
\text { Coefficient ( } \beta \text { ) }\end{array}$} & \multirow[t]{2}{*}{$\begin{array}{l}\text { Odds Ratio } \\
\text { (OR) }\end{array}$} & \multirow[t]{2}{*}{$p$-value } & \multicolumn{2}{|c|}{$\begin{array}{c}\text { Odds Ratio (OR) } \\
95 \% \text { Confidence Interval }\end{array}$} \\
\hline & & & & Lower Limit & Upper Limit \\
\hline \multicolumn{6}{|l|}{ GPA } \\
\hline$\geq B-$ & Ref. & Ref. & & & \\
\hline$<\mathrm{B}-$ & 1.132 & 3.102 & $<0.001^{*}$ & 1.994 & 4.825 \\
\hline \multicolumn{6}{|c|}{ Diagnosed with/treated for anxiety } \\
\hline No & Ref. & Ref. & & & \\
\hline Yes & 0.503 & 1.654 & $0.003^{*}$ & 1.189 & 2.300 \\
\hline \multicolumn{6}{|l|}{ Sleep difficulties } \\
\hline No & Ref. & Ref. & & & \\
\hline Yes & 0.570 & 1.768 & $<0.001^{*}$ & 1.306 & 2.395 \\
\hline \multicolumn{6}{|l|}{ Feeling hopeless } \\
\hline No & Ref. & Ref. & & & \\
\hline Yes & 0.723 & 2.061 & $<0.001^{*}$ & 1.453 & 2.925 \\
\hline \multicolumn{6}{|c|}{ Negative perceptions of body image } \\
\hline No & Ref. & Ref. & & & \\
\hline Yes & 0.346 & 1.413 & $0.031^{*}$ & 1.032 & 1.936 \\
\hline \multicolumn{6}{|l|}{ General health } \\
\hline Above average & Ref. & Ref. & & & \\
\hline Average & 0.406 & 1.501 & $0.013^{*}$ & 1.090 & 2.067 \\
\hline Below average & 0.588 & 1.800 & $0.008^{*}$ & 1.163 & 2.788 \\
\hline \multicolumn{6}{|c|}{ Managing daily responsibilities } \\
\hline Less than half the time & Ref. & Ref. & & & \\
\hline More than half the time & -0.576 & 0.562 & $<0.001^{*}$ & 0.414 & 0.762 \\
\hline
\end{tabular}

Note: ${ }^{*} p \leq 0.05$ 
the influence of anxiety on students' academic performance. This is followed by mentioning study limitations and proposing future research directions to address this important topic.

\section{Modifiable Factors of Interest}

Modifiable factors in the multivariable model with the greatest strength of association with the outcome variable are recognized as critical target areas warranting attention in informing program design and delivery on post-secondary campuses to attenuate the debilitative effects of anxiety on students' academic performance. Respondents to the NCHA-II survey who felt hopeless significantly increased their odds of reporting anxiety negatively affecting their grade point average (GPA) in this study. The only modifiable protective factor that significantly decreased the odds of the outcome variable include students who were able to effectively manage their daily responsibilities. Programs addressing these factors can instill hope, enhance self-efficacy, and build coping strategies to exert control in students' lives that benefit their mental health, academic performance, and overall university experience.

Snyder et al. (2002) posit hope as a cognitive process focusing on the ability of individuals to conceptualize goals, create a strategy to achieve their goals, and continually motivate themselves to execute and sustain their plan. Individuals who have high hope are more likely to attain their goals than those with lower levels of hope and are better able to modify their strategies to achieve their goals in the presence of barriers (Snyder et al., 2002). In the context of higher education relating to academic performance, students with hopeful attitudes are able to formulate specific, measurable, achievable, realistic, timely goals, as well as to motivate themselves to initiate and sustain study habits conducive to achieving their defined learning goals. A negative association exists between hope and anxiety, where individuals with low levels of hope experience more anxiety (Onwuegbuzie \& Snyder, 2000; Snyder, 1999), while a positive relationship is found between hope and students' GPA (Buckelew et al., 2008; Feldman \& Kubota, 2015; Rand et al., 2011). This is noteworthy as anxiety impairs students' goal-directed attention during educational assessments, in which they feel hopeless, become distracted by intrusive, self-deprecatory, goal-blocking thoughts, and exhibit negative emotions that adversely impact their academic performance (Conti, 2000; Snyder, 1999). Hope-based interventions have been found to improve levels of hope while reducing symptoms of anxiety (Cheavens et al., 2006). Programs that focus on building hope should be embedded at post-secondary institutions to reduce anxiety and support academic gains in students' lives (Seirup \& Rose, 2011).

Students who reported feeling adept at managing the responsibilities of daily life witnessed significant protective effects of anxiety not impacting their academics. This variable is a proxy measure of Ryff's (1989) environmental mastery, which is a psychological well-being construct indicative of how competent individuals are in exerting control over their life to manage and achieve goals. Individuals who score high levels of environmental mastery experience more confidence, improved purpose in life, and increased general life satisfaction amidst adversity (Ryff, 1989; September et al., 2001; Windle \& Woods, 2004). Perron (2006) found a reduction in this construct causes individuals to feel overwhelmed. Our study's results corroborate with these findings by suggesting the utility of strengthening environmental mastery can provide students with the confidence to have more control over their life to help undermine the implications of anxiety on their academic success. Time management is one item of the environmental mastery subscale that can be specifically targeted at post-secondary institutions to improve control over their life to successfully complete academic goal-directed activities (Claessens et al., 2007). Claessens et al.'s (2007) literature review provides a detailed account of the behaviours essential to fostering effective time management, including time assessment behaviours, planning behaviours, and monitoring behaviours. Briefly, time assessment behaviours aim for individuals to be conscientious of their use of time to ensure they have the capacity to achieve their goals (e.g., Claessens et al., 2007). Planning behaviours focus on utilizing time effectively by setting goals, planning tasks, and creating to-do lists (e.g., Claessens et al., 2007; Macan, 1996). Monitoring behaviours comprise individuals observing their use of time while completing tasks to create a feed back loop that limits distractions to ensure their goal is achieved (e.g., Claessens et al., 2007). Students who have strong time management skills exhibit higher levels of self-efficacy, motivation, and self-regulation in their learning, where they, in turn, are able to perform more optimally on academic assess- 
ments than their counterparts (e.g., Altun \& Erden, 2013; Indreica et al., 2011). Kelly (2003) also found that individuals' mental health can benefit from organizing time with structure and purpose, as it decreases feelings of worry. Since anxious individuals experience worrisome thoughts that impair their cognitive function, they can instead target the various aforementioned components of time management to attenuate the implications of anxiety, namely on their academic success. Students who engage in poor time management behaviours are more likely to procrastinate, experience test anxiety, and perform worse academically (Sansgiry \& Sail, 2006), further justifying why addressing poor time management is important. Time management skills can be challenging to cultivate, thereby making them essential to be taught on campus and promoted among post-secondary students to reduce anxiety in an academic setting (Jackson, 2009).

\section{Study Limitations}

There are various biases present within the data used for this project. First, there is a survival bias, as only current students registered at the institution were invited to participate in the NCHA-Il survey. Students who have dropped out due to overwhelming anxiety or poor academic performance may have been underrepresented in this dataset, resulting in a potential bias toward the null. A non-response bias may also create a limitation in this dataset. Students experiencing anxiety that impacted their academic performance may have been too overwhelmed to complete the survey, so they would be underrepresented in the analysis. In contrast, these individuals may have been more willing to procrastinate their academic obligations and complete the survey, resulting in an over-representation of this demographic. The NCHA-II data is also self-reported, which introduces some uncertainty about the truthfulness of responses. It is important to recognize the value of self-report surveys, as it can be challenging to obtain data regarding personal health habits and behaviours, specifically involving mental health, using alternate approaches (Foley et al., 2005). The respondents in the survey were predominantly female, which could emphasize risk and protective factors that are more commonly experienced by females. Finally, this study was interested in the effects of anxiety that had any impact on students' academic performance, where all academic implications were grouped together (e.g., receiving a lower grade on an exam was equal to experiencing significant disruption to one's thesis). Future research could investigate the factors influencing each of these individual options.

\section{Future Research Directions}

This paper utilized cross-sectional data to determine associations between various factors associated with the reporting of anxiety impacting students' academic performance, and concentrated on psychological variables that are modifiable using a proactive approach. Future longitudinal research should be conducted to confirm the direction of these associations to better understand how to prevent anxiety and other mental health issues, as well as to better understand how a university environment can be conducive to mitigating the implications of anxiety on students' academic success by instilling hope and fostering time management skill-building.

This research also supports the need for institutions to think beyond traditional targets of health promotion on campus. Post-secondary public health initiatives typically focus on the importance of exercise, diet, and sleep in students' lives. However, this study's results demonstrate the importance of providing tools focusing on the psychological level to enhance students' resiliency skills, which is often omitted from campus-wide initiatives. Future work could examine the role of additional psychological, attitudinal, and behavioural characteristics (e.g., grit, self-regulation, optimism, and happiness) in looking at the relationship between anxiety and academic performance. This can help provide a clearer picture of the true landscape of factors impacting anxious students at the post-secondary level, where future efforts or initiatives could be targeted on campuses. It is also suggested that future research should implement and evaluate the posed strategies to promote long-term sustainability.

This work also highlights the importance of having institutions better understand their campuses, as the demographics may require programs be amended to ensure the population is targeted appropriately, in which a one-size-fits-all approach may not be appropriate for all audiences. This work was limited in its ability to investigate students belonging to a non-binary gender or minority sub-population due to the small sample sizes, thereby highlighting the need to further research on the experiences of minority sub-populations (e.g., gender non-conforming populations, ethnic minorities, and 
students with developmental or learning disabilities). Such groups likely face unique challenges to their mental health, and could benefit from proactive programs to support their education and well-being.

\section{Acknowledgements}

We want to thank Shannon Thom for translating our abstract in French. Additionally, we are grateful that the American College Health Association (ACHA) captured and shared this data with our research team.

\section{Disclaimer}

The opinions, findings, and conclusions reported in this article are those of the authors and are in no way meant to represent the corporate opinions, views, or policies of the ACHA. ACHA does not warrant nor assume any liability or responsibility for the accuracy, completeness, or usefulness of any information presented in this article/ presentation.

\section{References}

Adams, T. B., Wharton, C. M., Quilter, L., \& Hirsch, T. (2008). The association between mental health and acute infectious illness among a national sample of 18- to 24-year-old college students. Journal of American College Health, 56(6), 657-664. https:/l doi.org/10.3200/JACH.56.6.657-664

Afolayan, J. A., Donald, B., Onasoga, O., Babafemi, A., \& Agama Juan, A. (2013). Relationship between anxiety and academic performance of nursing students, Niger Delta University, Bayelsa State, Nigeria. Advances in Applied Science Research, 4(4), 25-33. https://www.imedpub.com/articles/ relationship-between-anxiety-and-academic-performance-of-nursing-studentsniger-delta-university-bayelsa-state-nigeria.pdf

Altun, S., \& Erden, M. (2013). Self-regulation based learning strategies and self-efficacy perceptions as predictors of male and female students' mathematics achievement. Procedia-Social and Behavioral Sciences, 106, 2354-2364. https://doi.org/10.1016/j. sbspro.2013.12.270
American College Health Association. (2013a). American College Health Association-National College Health Assessment II: Canadian reference group date report spring 2013. American College Health Association. https://www.acha.org/documents/nchal ACHA-NCHA-II CANADIAN ReferenceGroup DataReport Spring2013.pdf

American College Health Association. (2013b). American College Health Association-National College Health Assessment II: Reliability and validity analyses 2011. American College Health Association. https://www. acha.org/documents/NCHA/ACHA-NCHAll RELIABILITY AND VALIDITY ANALYSES.pdf

American College Health Association. (2015). American College Health Association National College Health Assessment survey. https://www.acha.org/NCHA/ About ACHA NCHA/Survey/NCHA/About/Survey. aspx

American College Health Association. (2019). American College Health Association-National College Health Assessment II: Canadian reference group date report spring 2019. American College Health Association. https://www.cacuss.ca/files/Research/NCHA-II\%20 SPRING\%202019\%20CANADIAN\%20REFERENCE\%20GROUP\%20DATA\%20REPORT.pdf

Arnett, J. J. (2004). Emerging adulthood: The winding road from the late teens through the twenties. Oxford University Press.

Barrera, T. L., \& Norton, P. J. (2009). Quality of life impairment in generalized anxiety disorder, social phobia, and panic disorder. Journal of Anxiety Disorders, 23(8), 1086-1090. https://doi.org/10.1016/j. janxdis.2009.07.011

Baum, S., \& Ma, J. (2007). Education pays: The benefits of higher education for individuals and society (Trends in Higher Education Series). The College Board.

Beiter, R., Nash, R., McCrady, M., Rhoades, D., Linscomb, M., Clarahan, M., \& Sammut, S. (2015). The prevalence and correlates of depression, anxiety, and stress in a sample of college students. Journal of Affective Disorders, 173(1), 90-96. https://doi. org/10.1016/j.jad.2014.10.054 
Bellinger, D. B., DeCaro, M. S., \& Ralston, P. A. (2015). Mindfulness, anxiety, and high-stakes mathematics performance in the laboratory and classroom. Consciousness and Cognition, 37, 123-132. https://doi. org/10.1016/j.concog.2015.09.001

Böke, B. N., Mills, D. J., Mettler, J., \& Heath, N. L. (2019). Stress and coping patterns of university students. Journal of College Student Development, 60(1), 85-103. https://doi.org/10.1353/ csd.2019.0005

Brown, S. D., Tramayne, S., Hoxha, D., Telander, K., Fan, X., \& Lent, R. W. (2008). Social cognitive predictors of college students' academic performance and persistence: A meta-analytic path analysis. Journal of Vocational Behavior, 72(3), 298-308. https://doi.org/10.1016/j.jvb.2007.09.003

Buckelew, S. P., Crittendon, R. S., Butkovic, J. D., Price, K. B., \& Hurst, M. (2008). Hope as a predictor of academic performance. Psychological Reports, 103(2), 411-414. https://doi.org/10.2466/ pr0.103.2.411-414

Center for Collegiate Mental Health. (2018, January). 2017 Annual Report (Publication No. STA 18-166). https://sites.psu.edu/ccmh/files/2018/01/2017 CCMH Report-1r3iri4.pdf

Cheavens, J. S., Feldman, D. B., Gum, A., Michael, S. T., \& Snyder, C. R. (2006). Hope therapy in a community sample: A pilot investigation. Social Indicators Research, 77(1), 61-78. https://doi.org/doi:\%20 10.1007/s11205-005-5553-0

Chemers, M. M., Hu, L. T., \& Garcia, B. F. (2001). Academic self-efficacy and first year college student performance and adjustment. Journal of Educational Psychology, 93(1), 55-64. https://doi. org/10.1037/0022-0663.93.1.55

Claessens, B. J., Van Eerde, W., Rutte, C. G., \& Roe, R. A. (2007). A review of the time management literature. Personnel Review, 36(2), 255-276. https:/l pdfs.semanticscholar.org/0a2d/8a302693689ee3a3e444dc3204fbdd7bc6cc.pdf

Conti, R. (2000). College goals: Do self-determined and carefully considered goals predict intrinsic motivation, academic performance, and adjust- ment during the first semester? Social Psychology of Education, 4(2), 189-211. https://doi. org/10.1023/A:1009607907509

Cornish, P. A., Berry, G., Benton, S., Barros-Gomes, P., Johnson, D., Ginsburg, R., Whelan, B., Fawcett, E., \& Romano, V. (2017). Meeting the mental health needs of today's college student: Reinventing services through Stepped Care 2.0. Psychological Services, 14(4), 428-442. https://doi.org/10.1037/ $\underline{\text { ser0000158 }}$

Corona, R., Rodríguez, V. M., McDonald, S. E., Velazquez, E., Rodríguez, A., \& Fuentes, V. E. (2017). Associations between cultural stressors, cultural values, and Latina/o college students' mental health. Journal of Youth and Adolescence, 46(1), 63-77. https://doi.org/10.1007/s10964-016-0600-5

Curcio, G., Ferrara, M., \& De Gennaro, L. (2006). Sleep loss, learning capacity and academic performance. Sleep Medicine Reviews, 10(5), 323-337. https:/l doi.org/10.1016/j.smrv.2005.11.001

Dahl, R. E., \& Lewin, D. S. (2002). Pathways to adolescent health sleep regulation and behavior. Journal of Adolescent Health, 31(6), 175-184. https://doi. org/10.1016/S1054-139X(02)00506-2

Dohoo, I. R., Martin, W., \& Stryhn, H. (2009). Veterinary epidemiologic research (2nd ed.). VER Inc.

Dull, R. B., Schleifer, L. L., \& McMillan, J. J. (2015). Achievement goal theory: The relationship of accounting students' goal orientations with self-efficacy, anxiety, and achievement. Accounting Education, 24(2), 152-174. https://doi.org/10.1080/096392 84.2015.1036892

Eisenberg, D., Golberstein, E., \& Hunt, J. B. (2009). Mental health and academic success in college. The Berkeley Electronic Journal of Economic Analysis and Policy, 9(1), 1-35. https://doi.org/10.2202/1935$\underline{1682.2191}$

Eisenberg, D., Gollust, S. E., Golberstein, E., \& Hefner, J. L. (2007). Prevalence and correlates of depression, anxiety, and suicidality among university students. American Journal of Orthopsychiatry, 77(4), 534-542. https://doi.org/10.1037/0002$\underline{9432.77 .4 .534}$ 
Esmaeelzadeh, S., Moraros, J., Thorpe, L., \& Bird, Y. (2018). The association between depression, anxiety and substance use among Canadian post-secondary students. Neuropsychiatric Disease and Treatment, 14, 3241-3251. https://doi.org/10.2147/ NDT.S187419

Evidence Exchange Network. (2016). Transition-age youth evidence brief: Mental health promotion, prevention, and early intervention through campus interventions and integrated service centres. http://eenet.ca/sites/default/files/pdfs/TAYEnglish EENetEvidenceBrief Final.pdf

Eysenck, M. W., \& Derakshan, N. (2011). New perspectives in attentional control theory. Personality and Individual Differences, 50(7), 955-960. https://doi. org/10.1016/j.paid.2010.08.019

Eysenck, M. W., Derakshan, N., Santos, R., \& Calvo, M. G. (2007). Anxiety and cognitive performance: Attentional control theory. Emotion, 7(2), 336. https:// doi.org/10.1037/1528-3542.7.2.336

Farrer, L. M., Gulliver, A., Bennett, K., Fassnacht, D. B., \& Griffiths, K. M. (2016). Demographic and psychosocial predictors of major depression and generalised anxiety disorder in Australian university students. BMC Psychiatry, 16(1), 241. https://doi. org/10.1186/s12888-016-0961-Z

Feldman, D. B., \& Kubota, M. (2015). Hope, self-efficacy, optimism, and academic achievement: Distinguishing constructs and levels of specificity in predicting college grade-point average. Learning and Individual Differences, 37, 210-216. https://doi. org/10.1016/j.lindif.2014.11.022

Foley, K. L., Manuel, J., \& Vitolins, M. (2005). The utility of self-report in medical outcomes research. Evidence-Based Healthcare and Public Health, 9(3), 263-264. https://doi.org/10.1016/j.ehbc.2005.03.032

Glauser, W. (2017). Postsecondary campuses responding to record anxiety and depression levels. Canadian Medical Association Journal, 189(48), E1501-1502. https://doi.org/10.1503/cmaj.109-5512

Goswami, S., Sachdeva, S., \& Sachdeva, R. (2012). Body image satisfaction among female college students. Industrial Psychiatry Journal, 21(2), 168-172. https://doi.org/10.4103/0972-6748.119653

Hartmann, M. E., \& Prichard, J. R. (2018). Calculating the contribution of sleep problems to undergraduates' academic success. Sleep Health, 4(5), 463-471. https://doi.org/10.1016/j.sleh.2018.07.002

Hunt, J., \& Eisenberg, D. (2010). Mental health problems and help-seeking behavior among college students. Journal of Adolescent Health, 46(1), 3-10. https:ll doi.org/10.1016/j.jadohealth.2009.08.008

Indreica, E. S., Cazan, A. M., \& Truta, C. (2011). Effects of learning styles and time management on academic achievement. Procedia-Social and Behavioral Sciences, 30, 1096-1102. https://doi. org/10.1016/j.sbspro.2011.10.214

Jackson, V. P. (2009). Time management: A realistic approach. Journal of the American College of Radiology, 6(6), 434-436. https://doi.org/10.1016/j. jacr.2008.11.018

Kelly, W. E. (2003). No time to worry: The relationship between worry, time structure, and time management. Personality and Individual Differences, 35(5), 1119-1126. https://doi.org/10.1016/S01918869(02)00322-7

Kitzrow, M. A. (2009). The mental health needs of today's college students: Challenges and recommendations. NASPA Journal, 46(4), 646-660. https://doi. org/10.2202/1949-6605.1310

Kostanski, M., \& Gullone, E. (1998). Adolescent body image dissatisfaction: Relationships with self-esteem, anxiety, and depression controlling for body mass. The Journal of Child Psychology and Psychiatry and Allied Disciplines, 39(2), 255-262. https://doi. org/10.1111/1469-7610.00319

Krick, J. P., \& Sobal, J. (1990). Relationships between health protective behaviors. Journal of Community Health, 15(1), 19-34. https://doi.org/10.1007/ BF01350183

Larson, M., Orr, M., \& Warne, D. (2016). Using student health data to understand and promote academic success in higher education settings. College Student Journal, 50(4), 590-602. https://eric.ed.gov l?id=EJ1121548 
Lund, H. G., Reider, B. D., Whiting, A. B., \& Prichard, J. R. (2010). Sleep patterns and predictors of disturbed sleep in a large population of college students. Journal of Adolescent Health, 46(2), 124-132. https://doi.org/10.1016/j.jadohealth.2009.06.016

Macan, T. H. (1996). Time-management training: Effects on time behaviors, attitudes, and job performance. The Journal of Psychology, 130(3), 229-236. https:/l doi.org/10.1080/00223980.1996.9915004

MacKean, G. (2011). Mental health and well-being in post-secondary education settings: Pre-conference workshop. Canadian Association of College and University Student Services (CACUSS). https://citeseerx.ist.psu.edu/viewdoc/download?doi=10.1.1.737.6978\&rep=rep1\&type=pdf

Marcotte, J., \& Lévesque, G. (2018). Anxiety and well-being among students in a psychoeducation program: The mediating role of identity. Journal of College Student Development, 59(1), 90-104. https://doi.org/10.1353/csd.2018.0006

Merino, H., Senra, C., \& Ferreiro, F. (2016). Are worry and rumination specific pathways linking neuroticism and symptoms of anxiety and depression in patients with generalized anxiety disorder, major depressive disorder and mixed anxiety-depressive disorder? PloS One, 11(5), e0156169. https://doi. org/10.1371/journal.pone.0156169

Moylan, S., Maes, M., Wray, N. R., \& Berk, M. (2013). The neuroprogressive nature of major depressive disorder: Pathways to disease evolution and resistance, and therapeutic implications. Molecular Psychiatry, 18(5), 595-606. https://doi.org/10.1038/ mp.2012.33

Onwuegbuzie, A. J., \& Snyder, C. R. (2000). Relations between hope and graduate students' coping strategies for studying and examination-taking. Psychological Reports, 86(3), 803-806. https://doi. org/10.2466/pr0.2000.86.3.803

Orzech, K. M., Salafsky, D. B., \& Hamilton, L. A. (2011). The state of sleep among college students at a large public university. Journal of American College Health, 59(7), 612-619. https://doi.org/10.1080/0744 $\underline{8481.2010 .520051}$
Perron, B. E. (2006). A critical examination of the environmental mastery scale. Social Indicators Research, 79(1), 171-188. https://doi.org/10.1007/ $\underline{\text { s11205-005-4098-6 }}$

Rand, K. L., Martin, A. D., \& Shea, A. M. (2011). Hope, but not optimism, predicts academic performance of law students beyond previous academic achievement. Journal of Research in Personality, 45(6), 683-686. https://doi.org/10.1016/j.jp.2011.08.004

Ranđelović, D., Krstić, M., \& Babić-Antić, J. (2015). Academic success, anxiety and depressiveness as the predictors of life satisfaction among the students. Journal of Educational and Instructional Studies in the World, 5(1), 63-73. https://arastirmax.com/ tr/publication/journal-educational-and-instructional-studies-world/5/1/63-73-academic-success-anxiety-and-depressiveness-predictors-life-satisfaction-among-students/arid/d2591d8f-5016

Reavley, N., \& Jorm, A. F. (2010). Prevention and early intervention to improve mental health in higher education students: A review. Early Intervention in Psychiatry, 4(2), 132-142. https://doi.org/10.1111/ j.1751-7893.2010.00167.x

Rose, C., Godfrey, K., \& Rose, K. (2015). Supporting student wellness: De-stressing initiatives at memorial university libraries. Partnership: The Canadian Journal of Library and Information Practice and Research, 10(2), 1-21. https://research.library.mun. ca/11832/1/3564-19547-5-PB.pdf

Ryff, C. D. (1989). Happiness is everything, or is it? Explorations on the meaning of psychological well-being. Journal of Personality and Social Psychology, 57(6), 1069-1081. https://doi.org/10.1037/0022$\underline{3514.57 .6 .1069}$

Sansgiry, S. S., \& Sail, K. (2006). Effect of students' perceptions of course load on test anxiety. American Journal of Pharmaceutical Education, 70(2), 26. https://europepmc.org/article/med/17149404

Seirup, H., \& Rose, S. (2011). Exploring the effects of hope on GPA and retention among college undergraduate students on academic probation. Education Research International, 2011, 1-7. https://doi. org/10.1155/2011/381429 
September, A. N., McCarrey, M., Baranowsky, A., Parent, C., \& Schindler, D. (2001). The relation between well-being, impostor feelings, and gender role orientation among Canadian university students. The Journal of Social Psychology, 141(2), 218-232. https://doi.org/10.1080/00224540109600548

Snyder, C. R. (1999). Hope, goal blocking thoughts, and test-related anxieties. Psychological Reports, 84(1), 206-208. https://doi.org/10.2466/pr0.1999.84.1.206

Snyder, C. R., Shorey, H. S., Cheavens, J., Pulvers, K. M., Adams III, V. H., \& Wiklund, C. (2002). Hope and academic success in college. Journal of $E d-$ ucational Psychology, 94(4), 820-826. https://doi. org/10.1037/0022-0663.94.4.820

Spring, B., Moller, A. C., \& Coons, M. J. (2012). Multiple health behaviours: Overview and implications. Journal of Public Health, 34, i3-i10. https://doi. org/10.1093/pubmed/fdr111

StataCorp LLC. (2018). StataSE (Release 15.1) statistical software [Computer software]. StataCorp LLC.

Windle, G., \& Woods, R. T. (2004). Variations in subjective wellbeing: The mediating role of a psychological resource. Ageing \& Society, 24(4), 583-602. https:/l doi.org/10.1017/S0144686X04002107

Wyatt, T., \& Oswalt, S. B. (2013). Comparing mental health issues among undergraduate and graduate students. American Journal of Health Education, 44(2), 96-107. https://doi.org/10.1080/19325037.20 $\underline{13.764248}$

\section{Contact Information}

Konrad T. Lisnyj

klisnyj@uoguelph.ca 